\title{
Comment on epinephrine during resuscitation of traumatic cardiac arrest and increased mortality: a post hoc analysis of prospective observational study
}

\author{
Makoto Aoki ${ }^{1 *}$ and Toshikazu Abe ${ }^{2}$
}

\begin{abstract}
The aim of this Letter to the Editor was to report some important biases in a recently published Article. We agreed with the notion by Yamamoto et al. that the effects of epinephrine regarding was limited without hemostasis, however, this study had major limitations such as no information on etiology of traumatic cardiac arrest (hemorrhagic or on non-hemorrhagic) and on hemostatic treatment. The results of this study should be interpreted with caution and further analysis is necessary. Finally, we commented on the necessity of future study regarding another vasopressor (ie; vasopressin) on traumatic cardiac arrest based on current evidence.
\end{abstract}

Keywords: Epinephrine, Traumatic cardiac arrest, Biases, Vasopressin

\section{Dear Editor.}

We read with interest the article of Yamamoto, et al., who reported that epinephrine administration during in-hospital resuscitation of traumatic cardiac arrest was associated with increased mortality [1]. Yamamoto et al. [1] performed post-hoc data analysis on a prospective, multicentre, observational study (SOSKANTO 2012) consisting of patients who suffered outof-hospital cardiac arrest and were transported to 67 emergency hospitals by emergency medical services personnel. We agree with the notion by Yamamoto et al. that the effects of epinephrine regarding spontaneous circulation would not persist without hemostasis, and our previous investigation demonstrated that prehospital epinephrine administration was associated with increased temporal return of spontaneous circulation but was not associated with survival [2]. The study of Yamamoto et al. [1] demonstrated that administration of epinephrine was associated with increased mortality even in in-hospital situations where prompt hemostatic treatment could be deployed.

\footnotetext{
* Correspondence: aokimakoto@gunma-u.ac.jp

${ }^{1}$ Department of Emergency Medicine, Gunma University Graduate School of Medicine, Maebashi, Japan

Full list of author information is available at the end of the article
}

However, we have to interpret the findings of the study of Yamamoto et al. [1] while considering some major limitations. The most important limitation is that the main etiology of traumatic cardiac arrest in the subjects was not known in this study, that is, whether the etiology was hemorrhagic or nonhemorrhagic. Lin, et al. [3] showed that the effect of epinephrine on traumatic cardiac arrest in children differed depending on whether the etiology was hemorrhagic or non-hemorrhagic. Additionally, the details of hemostatic treatment such as emergency thoracotomy, resuscitative endovascular balloon occlusion of the aorta and time to treatment were not entered in the SOS-KANTO 2012 database. Inhospital procedures vary among hospitals and this bias could have affected the results.

Currently, vasopressin is the only vasopressor that has the possibility of improving the outcome of trauma patients [4]. A recent randomized controlled trial showed that administration of low-dose arginine vasopressin to hemorrhagic shock patients decreased blood product requirements [4]. No study has investigated the effect of administration of vasopressin on traumatic cardiac arrest; therefore, further trials are needed. 


\section{Acknowledgements}

Not applicable.

\section{Authors' contributions}

MA wrote the manuscript. TA revised the manuscript. Both authors had read and approved the final manuscript.

\section{Funding}

No funding.

\section{Availability of data and materials}

Data sharing not applicable to this article as no datasets were generated or analyzed during the current work.

\section{Ethics approval and consent to participate}

Not applicable.

\section{Consent for publication}

Not applicable.

\section{Competing interests}

The authors declare that they have no conflicts of interest.

\section{Author details}

'Department of Emergency Medicine, Gunma University Graduate School of Medicine, Maebashi, Japan. ${ }^{2}$ Department of General Medicine, Juntendo

University, Tokyo, Japan.

Received: 12 November 2019 Accepted: 19 November 2019

Published online: 27 November 2019

\section{References}

1. Yamamoto R, Suzuki M, Hayashida K, Yoshizawa J, Sakurai A, Kitamura N, et al. Epinephrine during resuscitation of traumatic cardiac arrest and increased mortality: a post hoc analysis of prospective observational study. Scand J Trauma Resusc Emerg Med. 2019;27(1):74.

2. Aoki M, Abe T, Oshima K. Association of prehospital epinephrine administration with survival among patients with traumatic cardiac arrest caused by traffic collisions. Sci Rep. 2019;9(1):9922.

3. Lin YR, Wu MH, Chen TY, Syue YJ, Yang MC, Lee TH, et al. Time to epinephrine treatment is associated with the risk of mortality in children who achieve sustained ROSC after traumatic out-of-hospital cardiac arrest. Crit Care. 2019;23(1):101.

4. Sims CA, Holena D, Kim P, Pascual J, Smith B, Martin N, et al. Effect of lowdose supplementation of arginine vasopressin on need for blood product transfusions in patients with trauma and hemorrhagic shock: a randomized clinical trial. JAMA Surg. 2019. https://doi.org/10.1001/jamasurg.2019.2884.

\section{Publisher's Note}

Springer Nature remains neutral with regard to jurisdictional claims in published maps and institutional affiliations. 